\title{
Energy expenditure rate in level and uphill treadmill walking determined from empirical models and foot inertial sensing data
}

\author{
A.M. Sabatini, C. Martelloni, S. Scapellato and F. Cavallo \\ Scuola Superiore Sant’Anna, Piazza Martiri della Liberta`33, 56127 Pisa, Italy \\ E-mail: a.sabatini@sssup.it
}

An empirical model is used for predicting the energy expenditure rate of treadmill walking from walking speed and incline, which are measured by a foot-mounted inertial sensor. The difference between values of the energy expenditure rate obtained by entering measured and true values of these variables in the model equation is less than the errors that are reported to affect model based assessments of the metabolic response to locomotion in humans.

Introduction: The quantification of energy expenditure rate (EER) is of great interest in a number of applications, including rehabilitation, disease prevention, fitness management and sport medicine. Several techniques are currently available to quantify EER in unrestrained conditions, including doubly labelled water, indirect calorimetry, hearth rate monitors, motion sensors [1]. Motion sensing, such as trunk accelerometry, has been proposed to find a method to evaluate the EER related to locomotion [2]. However, without an external measurement of the incline, the EER of uphill/downhill walking cannot be accurately predicted by standard methods of analysis of body accelerations [3]. As an alternative, motion sensing to determine both walking speed and incline has been proposed. The combined trunk-heel accelerometry described in [4] performs this measuring task by an indirect method based on artificial neural network processing. We are currently working on foot inertial sensing, a technique potentially capable of estimating walking speed and incline by direct methods based on strap-down integration [5]. In this Letter, we discuss the problem of estimating EER by entering the measured values of walking speed and incline into one of the many empirical models that are available to assess submaximal walking using a treadmill.

Empirical model: The American College of Sports Medicine (ACSM) has published an equation that estimates the oxygen cost of performing steady-state treadmill walking, which is assumed to be valid for speeds less than $6 \mathrm{~km}=\mathrm{h}(100 \mathrm{~m}=\min )$ [6]:

$$
\dot{E_{w}}=0.1 v+1.8 v+3.5
$$

where the EER $\dot{E_{\mathrm{w}}}$, the speed of walking $\mathrm{v}$ and the incline a are measured in $\mathrm{mlO}_{2} / \mathrm{kg} \mathrm{min}, \mathrm{m} / \mathrm{min}$ and $\%$ grade, respectively. Since several factors, including sex, age, body morphology and resting metabolism, are known to significantly affect $\ddot{\tilde{E}_{w}}$, the ACSM equation is helpful to get a gross indication of the metabolic expense incurred in treadmill walking.

Experimental setup: One biaxial accelerometer (Analog Devices, ADXL210E), and one rate gyroscope (Murata, ENC-03J) were embedded in the developed inertial measurement unit (IMU). The accelerometer and gyroscope sensitivity were $100 \mathrm{mV}=\mathrm{g}(\mathrm{g} 1 / 4$ $9.81 \mathrm{~m}=\mathrm{s} 2$ is the gravity acceleration) and $3 \mathrm{mV}=\mathrm{deg}=\mathrm{s}$, respectively. The sensor signals were sampled at $\mathrm{f}_{\mathrm{s}}{ }^{1} / 4200 \mathrm{~Hz}$ (12 bits) using a DAQ card-6062E (National Instruments, (NI)), which was controlled under NI's LabView 6. Matlab 6 was used for all subsequent data analysis tasks, including lowpass filtering by a doublepass second-order Butterworth filter with cutoff frequency at $15 \mathrm{~Hz}$. The IMU was placed on the dorsal aspect of the foot and attached snugly with a Velcro strap, so as to have the accelerometer sensitive axes in the sagittal plane; the gyroscope sensitive axis was oriented in the mediallateral direction by IMU construction, see Fig. 1. For each of the five healthy adultmales tested so far with the IMU, 1minlong data records were obtained from walking trials carried on a motorized treadmill (Runrace HC1200, Technogym, Italy) at several combinations of controlled speed and incline (seven walking speeds from 3 to $6 \mathrm{~km}=\mathrm{h}$ in steps of $0.5 \mathrm{~km}=\mathrm{h}$, at each of four different inclines: $0,5,10,15)$ - paper in preparation. Finally, one subject (height: $1.81 \mathrm{~m}$; weight: $88 \mathrm{~kg}$ ) was asked to perform the UPHILL treadmill programme, namely a walking trial lasting $9 \mathrm{~min}$, with speeds and inclines varying inside the interval $[5.5,6] \mathrm{km}=\mathrm{h}$ and $[0,11]$, respectively.

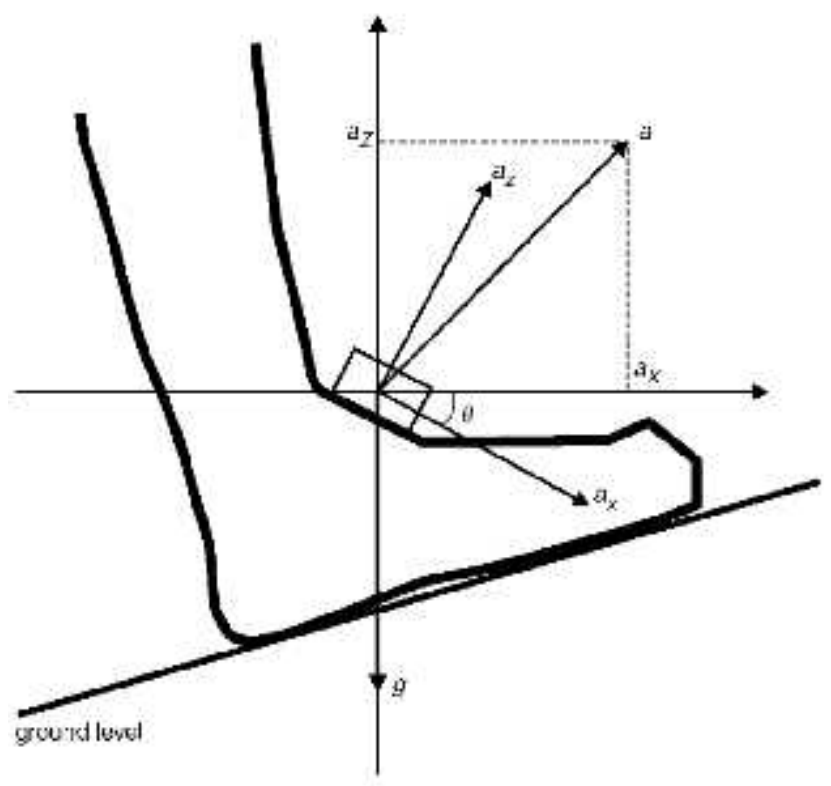


Fig. 1 Schematic representation of measurement setup. The biaxial accelerometer measures acceleration components ax, az, which include contributions from foot acceleration $\mathrm{a}=[\mathrm{ax}, \mathrm{az}]$ and gravitational acceleration $\mathrm{g}$. The foot gyroscope measures angular velocity around its sensitive axis, which is assumed to be orthogonal to the vertical plane, and allows reconstruction of inclination angle $\vartheta$ by integration.

Data processing: A foot contact detection algorithm was applied to the gyroscope signal for gait event identification, i.e. heel-off, toe-off, heel strike, foot-flat and estimation of the temporal gait parameters, including stride time and relative stance. By assuming a two-dimensional gait model, the foot acceleration was projected in the horizontal and vertical directions, and the corresponding velocity and distance components were obtained from single- and double-integration. The gyroscope was used to compensate for the rotation of the sensor case: the angular velocity was integrated to estimate the inclination of the foot relative to the ground - the pitch angle - so as to remove the gravitational acceleration from the accelerometer readings. Resetting mechanisms were built in the strap-down algorithm to deal with incorrect sensor nulling and accumulation of errors during integration; since the foot is almost steady during stance, initial conditions for the pitch angle were established by using the accelerometer as a (static) inclinometer, while null initial conditions were assumed for the velocity components.

The estimated spatial gait parameters, namely the stride length decomposed in its horizontal and vertical components, which are, respectively, perpendicular and parallel to gravity (see Fig. 1), were used to estimate the walking speed, in conjunction with the stride time, and the incline. These estimated values were further averaged over each 1 min-long data record to produce $\mathrm{Vm}$ and $\mathrm{am}$, to be entered into (1) for determining $E_{\mathrm{wm}}^{*}$. As for the UPHILL trial, the estimates of walking speed and incline, available at each gait cycle, were entered into (1) and then integrated over time to determine the energy expenditure.

Results: $\mathscr{E}_{w \mathrm{~m}}^{\cdot}$ differs from the value $\mathscr{E}_{\mathrm{wE}}^{\cdot}$, which is obtained by entering $\mathrm{v}$ and a into (1): the root mean square error is RMSE=2.121 $\mathrm{mlO}_{2} / \mathrm{kg}$ min, which originates from errors on both walking speed (RMSE $=0.136 \mathrm{~km} / \mathrm{h}$ ) and incline (RMSE $\left.=1.515 \%\right)$. Standard least squares regression tools are applied to the measured against predicted EER values, to yield: $E_{w \mathrm{~m}}^{*}=0.871 E_{w \mathrm{r}}^{*},+1.893, \mathrm{r} 2>0.99$; the regression standard error (SE) is $\mathrm{SE}=5.933 \mathrm{mlO} / \mathrm{kg} \mathrm{min}$. By discarding the data related to the steepest incline, we obtain:

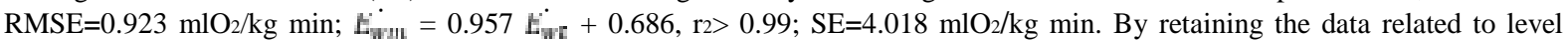

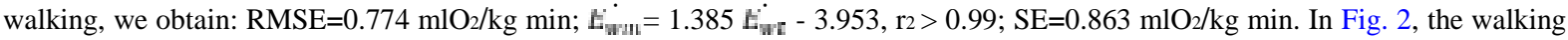
speed at different gait cycles are reported for the entire duration of the UPHILL programme. According to the UPHILL profile instructions, the energy expenditure is $E_{w}=85.14 \mathrm{kcal}$, which is obtained by integrating $\vec{E}_{\mathrm{w}}$ over the 9 min-long time interval ( $1 \mathrm{kcal}$ is, approximately, $200 \mathrm{mlO}_{2}$ ). In this particular run, the IMUderived estimate is $\mathrm{E}_{\mathrm{w}}=89.30 \mathrm{kcal}$ (relative error less than $5 \%$ ).

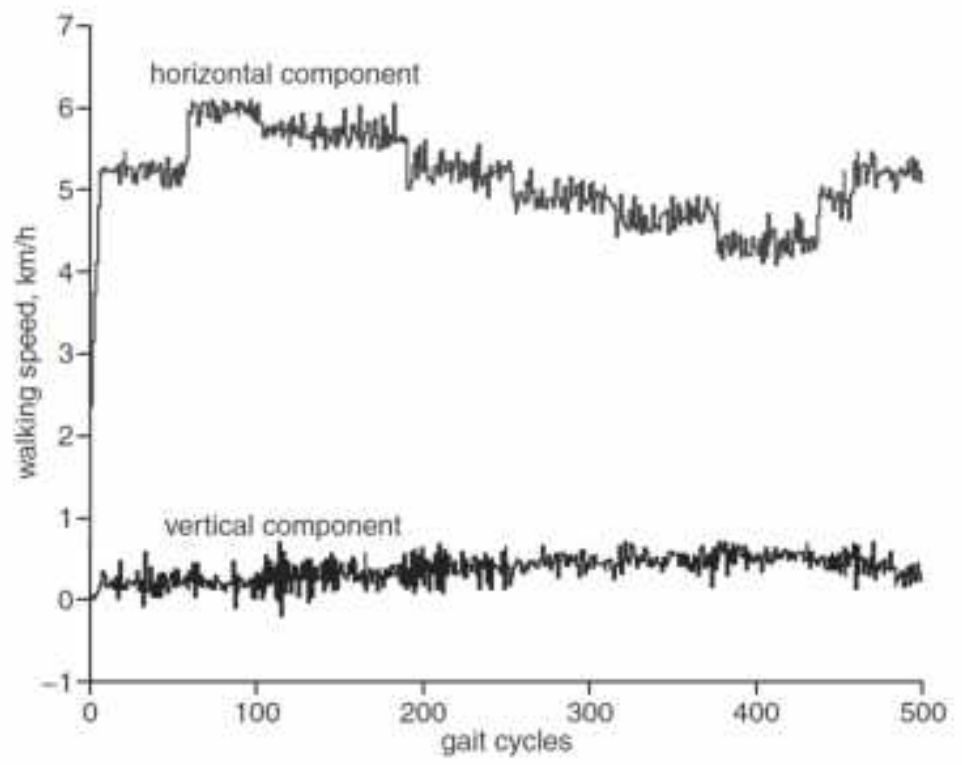

Fig. 2 Walking speed, decomposed in its estimated horizontal and vertical components, measured during UPHILL programme carried by one subject.

Discussion and conclusions: In our experimental data, it is noted that the IMU tends to slightly underestimate both walking speed and incline. This underestimation affects, in particular, the incline (notably, at the steepest incline tested), which also impacts the 'accuracy' of the EER determination. It is observed in (1) that the estimation error $\Delta \dot{E}_{w}$ must increase with measurement errors of both walking speed and incline. Nonetheless, the standard error affecting the linear relationship constructed between $\hat{E}_{\mathrm{wm}}^{\cdot}$ and $\hat{E}_{\mathrm{mi}}^{\circ}$ is less than the standard errors reported in [7]. Finally, the UPHILL test results allow us to state that, whatever is the meaning of energy expenditure when steady-state metabolic conditions are not given time to develop, the proposed approach can be used to perform long-term monitoring of walking in terms of 'burnt calories'. We have proposed a simple one-axis strap-down IMU to estimate the EER. This is done by using the measured walking speed and incline in the ACSM equation. The consequence of the inertial measurement errors are within the limits of physiological variability affecting the accuracy of an empirical model, which is claimed to have concurrent validity as for the assessment of the metabolic response (oxygen uptake) to steady-state submaximal treadmill walking in humans. In conclusion, foot inertial sensing is a promising tool for determining the energy expenditure of unrestrained walking, since accurate measurement of walking speed and incline is fundamental in all methods devised to this aim so far. 


\section{References}

1. Montoye, H.J., et al.: 'Movement assessment devices' in 'Measuring physical activity and energy expenditure' (Human Kinetics, Champaign, IL, USA, 1996),pp. 6-14.

2. Bouten, C.V.C., et al.: 'A triaxial accelerometer and portable data processing unit for the assessment of daily physical activity', IEEE Trans. Biomed. Eng., 1997, 44, (3), pp. 136-147.

3. Terrier, P., Aminian, K., and Schutz, Y.: 'Can accelerometry accurately predict the energy cost of uphill=downhill walking?', Ergonomics, 2001, 44, (1), pp. 48-62.

4. Aminian, K., et al.: 'Incline, speed, and distance assessment during unconstrained walking', Med. Sci. Sports Exerc., 1995, 27, pp. 226-234

5. Sabatini, A.M., Genovese, V., and Maini, E.S.: 'Portable system for data acquisition and transmission based on handheld PC technology', Electron. Lett., 2002, 38, (25), pp. 1635-1637.

6. American College of Sports Medicine: 'Guidelines for exercise testing and prescription' (Lea \& Febiger Co, Philadelphia, 1991, 2nd edn.), pp. 285-300

7. Bunc, V., and Dlouha': 'Energy cost of treadmill walking', J. Sports Med. Phys. Fitness, 1997, 37, pp. 103-109. 